\title{
SEMIOLOGIA NEUROLOGICA NUMA POPULAÇÃO DE ESCOLARES DA PRIMEIRA SÉRIE DO ENSINO FUNDAMENTAL
}

\author{
VANDA M. G. GONÇALVES*, JOSIANE M. F. TONELOTTO**, SOLANGE G. RAVANIN/***
}

\begin{abstract}
RESUMO - Avaliamos escolares de primeira série do ensino fundamental, utilizando semiologia neurológica. Foram convidados, sem o conhecimento prévio do desempenho escolar, todos os alunos que frequentavam 5 classes de primeira série do primeiro grau de uma escola pública escolhida ao acaso, no município de Itatiba / São Paulo, cujos pais assinaram o Termo de Consentimento Livre e Esclarecido. Foram excluídos aqueles cujos pais não assinaram esse termo ou não compareceram a 3 consultas agendadas em dias diferentes. Utilizou-se o Exame Neurológico Tradicional (ENT) (Lefèvre, 1972). Considerou-se como normal a realização de todas as provas do ENT e as medidas do perímetro craniano propostas por Diament \& Rodrigues (1976). Os dados foram armazenados em banco de dados do programa Epi6 (Epidemiologic Information). Os resultados foram analisados pelo cálculo de porcentagem e pelo teste $\chi^{2}$. O nível de significância foi 0,05. Foram avaliados 124 alunos. O ENT foi normal em $87(70,16 \%)$ e alterado em $37(29,83 \%)$ escolares. Entre as alterações, foram observados: leve tremor, leve hipotonia muscular, atraso na aquisição da fala, macrocefalia, microcefalia, hiperatividade, síndrome de nervo craniano e paresia facial central. Um escolar apresentou síndrome de liberação piramidal nos membros inferiores.
\end{abstract}

PALAVRAS-CHAVE: crianças, escolares, exame neurológico.

\section{Neurologic examination of a group of children attending first grade of a elementary school}

ABSTRACT - We evaluated children in the first grade of a elementary school using neurological examination. With no previous knowledgement of their educational performance, were invited all children attending five classes of the first grade of an elementary public school chosen randomly, in Itatiba / Sao Paulo / Brazil, whose parents assigned a Commitment Term for participation in this research. Children who missed three evaluations in different days or whose parents did not assigned the Commitment Term were excluded. The Traditional Neurological Examination (ENT) (Lefevre, 1972) was applied. It was considered for normal the measurement of the skull circumference, proposed by Diament \& Rodrigues (1976), and the application of all ENT items. The data were stored in a database of the Epi6 Program (Epidemiologic Information), and analyzed by percentage calculation and by the $\chi^{2}$ test. The significance level was 0.05 . Children evaluated were 124 . The ENT results were normal in 87 $(70.16 \%)$ and altered in $37(29.83 \%)$. Among the alterations, there were observed: light tremor, light muscular hypotonia, speech acquisition delay, macrocephaly, microcephaly, hyperactivity, cranial nerve syndrome, central facial paralysis. One child presented corticospinal tract impairment syndrome of the distal lower extremities.

KEY WORDS: elementary school children, neurological examination

Desde o nascimento, o cérebro está em constante evolução, através de sua inter-relação com o meio. A criança percebe o mundo pelos sentidos, age sobre ele e esta interação se modifica durante a evolução, entendendo melhor, pensando de modo mais complexo, comportando-se com maior precisão

Departamento de Neurologia, Faculdade de Ciências Médicas (FCM) da Universidade Estadual de Campinas (UNICAMP): *Professor Assistente Doutor, Coordenador da Área de Concentração em Neurologia do Curso de Pós-graduação em Ciências Médicas da FCM/UNICAMP; ** Doutor em Ciências Médicas, Área de Concentração em Neurologia/FCM/UNICAMP, Psicóloga, Diretora do Instituto de PG Lato Sensu e Extensão/ Universidade São Francisco; *** Mestre em Ciências Médicas, Área de Concentração em Neurologia/FCM/ UNICAMP, Fisioterapeuta, CEPRE "Gabriel Porto". Pesquisa apoiada em parte pela Fundação de Amparo à Pesquisa do Estado de São Paulo (FAPESP). Aceite: 18-novembro-1999.

Dra. Vanda M. G. Gonçalves - Departamento de Neurologia FCM/UNICAMP - Caixa Postal 6111 - 13 083-970 Campinas SP - Brasil. E mail: vanda@bestway.com.br 
à medida que controla mais seu corpo e elabora mais corretamente suas idéias ${ }^{1}$. Esse é o processo de aprendizagem, o qual se faz no sistema nervoso central (SNC), onde se produzem mudanças mais ou menos permanentes, que se traduzem por uma modificação funcional ou condutual que permite melhor adaptação do indivíduo a seu meio, como resposta a uma ação ambiental ${ }^{2}$. O processo de aprender deve levar a criança a realizar suas atividades de interpretação e de reprodução, partindo de uma função cerebral gnósica-interpretativa e práxico-produtiva, mostrando que aprender é uma função cognitiva e não uma simples cópia de atitudes sem significado ${ }^{3}$. As aprendizagens formais transmitidas pela escola, essencialmente dependem, para que sejam processadas, da integração do pensar, sentir, falar, ouvir e agir, influenciados pela ação constante de fatores internos e externos ao indivíduo, quer sejam eles de origem orgânica, cognitiva e emocional (internos), quer sejam de origem social e pedagógica (externos) ${ }^{4}$. A educação denominada escolar, apesar do interesse e de ter sido melhor estudada por outras disciplinas, passou a despertar o interesse de psicólogos educacionais, a partir dos anos cinquenta, quando da necessidade de se estudar melhor o processo ensino/aprendizagem 5 . O motivo pelo qual tantos especialistas se preocupam com o assunto, deve-se ao fato de que o sucesso do indivíduo na sociedade está inteiramente ligado à sua capacidade para aprender ${ }^{2}$. É senso comum dizer que os problemas de escolaridade são definidos pela incapacidade apresentada por um aluno de cumprir os conteúdos mínimos definidos como padrão para o seu grupo, independente de ser esta incapacidade causada por fatores internos ou externos ${ }^{6}$. Quando uma criança apresenta problemas para aprender, eles podem ser investigados de diferentes formas e com metodologias distintas, com alguns teóricos defendendo a idéia que eles podem ser mais atribuídos a fatores internos e outros que os atribuem aos fatores externos ${ }^{7}$.

A queixa de distúrbio de aprendizagem, seja principal ou secundária ${ }^{8}$, não se baseia em nada sistemático e normalmente, a escola que encaminha a criança para avaliação o faz de forma aleatória, pelo simples fato da criança não corresponder aos padrões do ensino regular.

No processo avaliativo de crianças com dificuldades escolares ${ }^{8}$, chama atenção o ponto fundamental no diagnóstico: a criança é retirada do processo de ensino na sala de aula, levada ao consultório, retornando à sala de aula sem soluções, mas com maior aceitabilidade do meio pelo fato de ter-se provado que ela não tem nada na "cabeça".

A distinção tênue entre os processos neurológicos e psicológicos, exigem um diagnóstico que seja preciso em informar sobre o padrão normal da aprendizagem ou alteração nesse padrão ${ }^{9}, o$ que leva a criança a receber, internalizar e relacionar o conteúdo a ser aprendido, com um estilo próprio e independente do programa de ensino onde esteja inserida. O processo diagnóstico em si, por sua vez, também deve ser melhor preparado ${ }^{9}$, eliminando-se provas, unificando-se procedimentos que sejam capazes de, no menor espaço de tempo possível, categorizar crianças com e sem alterações.

Educadores, médicos, profissionais da área de saúde, pais, solicitam avaliações neurológicas, com a finalidade de encontrar a explicação para a dificuldade escolar na infância. Na maioria das vezes, o resultado é normal ou são encontradas discretas alterações, que não explicam a dificuldade em aprender. Poderiam estas discretas alterações ser encontradas em crianças sem problemas de aprendizagem?

Com o objetivo de conhecer aspectos neurológicos de um grupo de crianças frequentando a primeira série do primeiro grau, sem o conhecimento prévio do desempenho escolar delas, foi proposto este estudo, no sentido de se avaliar padrões neurológicos individuais, independentemente de cumprirem ou não os conteúdos escolares propostos.

\section{METODO}

Foram incluídos todos os alunos que frequentavam, no ano de 1998, cinco classes da primeira série do primeiro grau de uma escola pública escolhida ao acaso, sendo realizado um estudo piloto no município de Itatiba / São Paulo. Não houve conhecimento prévio do rendimento escolar dos alunos.

Foram enviados 145 Termos de Consentimento Livre e Esclarecido e devolvidos a tempo e devidamente preenchidos, 130. Foram excluídos outros 6 escolares que faltaram à avaliação agendada em três datas diferentes. Assim, foram consideradas a amostra da pesquisa, um total de 124 escolares. 
A faixa etária variou entre 6 anos e 10 meses e 9 anos e 4 meses. Foram do sexo masculino, 68 (54,83\%) e do sexo feminino, $56(45,16 \%)$.

Os escolares foram avaliados numa sala medindo 4x6 metros, cedida pela diretoria da escola. Cada criança foi avaliada individualmente por um neurologista infantil.

Utilizou-se o Exame Neurológico Tradicional $(\mathrm{ENT})^{10}$, que avalia crânio, fala, força muscular, tono muscular (através da palpação, movimento passivo e balanço passivo dos membros superiores e inferiores); reflexos profundos e superficiais; equilíbrio estático e dinâmico, coordenação apendicular, coordenação troncomembros, sensibilidade, nervos cranianos e sinais meníngeos.

Considerou-se como normal as medidas do perímetro craniano variando no sexo masculino, entre 49,0 e 54,5 centímetros (cm) aos 7 anos; 49,5 e 55,0 cm aos 8 anos e entre 50,0 e 55,5 cm aos 9 anos; variando no sexo feminino, entre 49,0 e 53,5 cm aos 7 anos; 49,0 e $54,0 \mathrm{~cm}$ aos 8 anos e entre 49,5 e 54,5 cm aos 9 anos ${ }^{11}$.

Considerou-se como padrão neurológico normal a realização de todas as provas do ENT e como alteração a anormalidade de respostas em qualquer das provas.

Os resultados foram armazenados em banco de dados do programa Epi6 (Epidemiologic Information). Concomitante com a execução das manobras, os dados coletados foram imediatamente digitados por dois auxiliares de pesquisa, sendo um fiscal. Após cada avaliação neurológica, antes da dispensa do escolar, os dados foram revisados e conferidos. Utilizou-se o Notebook Intel Inside 386, cedido para este projeto pelo Serviço de Recursos Audiovisuais da Universidade Estadual de Campinas (Unicamp). Os resultados foram analisados pelo cálculo de porcentagem e pelo teste $\chi^{2}$. O nível de significância foi 0,05 .

\section{RESULTADOS}

O ENT foi normal em 87 (70,16\%) e alterado em 37 (29,83\%) escolares, identificados na Tabela 1. Não houve associação significativa quanto ao sexo $\left(\chi^{2}=0,08 ; \mathrm{p}=0,77\right)$.

Entre os que apresentaram semiologia neurológica alterada, verificou-se uma única alteração em 31 (25,00\%) crianças. Apresentaram duas alterações, 6 (4,83\%) escolares (Casos 16, 18, 49, 51, 123 e 127). Nestes foi observada associação de paresia facial central direita e síndrome de VI nervo craniano à direita; macrocefalia associada a leve hipotonia muscular; atraso na aquisição da fala, clono de pés ou hipotonia muscular de membros associados a leve tremor. Uma criança apresentou três alterações neurológicas, (Caso 60), constituídas por leve hipotonia muscular nos membros, reflexos aquilianos exaltados e clono de pés esgotáveis e simétrico.

$\mathrm{Na}$ avaliação do crânio, apresentaram alterações na palpação 9 (7,25\%) escolares, sendo observado: batente óssea bregmática ou metópica em 5 (Casos 7, 13, 16, 36 e 57); exostose em 3 (Casos 37, 108 e 129) e achatamento da região occipital em 1 (Caso 118).

Tabela 1. Identificação dos achados no Exame Neurológico Tradicional.

\begin{tabular}{ll}
\hline Alteração & $\mathrm{N}$ \\
\hline Dislalia & Casos $30,34,42,78,81,84,123$ \\
Microcefalia & Caso 12 \\
Macrocefalia & Casos $6,51,83,121$ \\
Hiperatividade & Caso 113 \\
Hipotonia leve & Casos $10,16,38,39,48,49,51,60,62,80,96,97,102,125,129$ \\
Ref. Assimétrico & Caso 3 \\
Clono bilateral & Casos 60,127 \\
Tremor leve & Casos $8,16,28,33,47,59,115,117,122,123,127$ \\
Nervo craniano & Caso 18 \\
\hline
\end{tabular}

$\mathrm{N}$, Identificação do número do sujeito. 
Tabela 2. Distribuição da amplitude do balanço passivo segundo o sexo.

\begin{tabular}{lccc}
\hline Sexo & Balanço Passivo Normal & Balanço Passivo Aumentado & Total \\
\hline Feminino & 22 & 34 & 56 \\
Masculino & 21 & 47 & 68 \\
Total & 43 & 81 & 124 \\
\hline
\end{tabular}

$\chi^{2,} 0,96 ; \mathrm{p}, 0,32$.

O perímetro craniano (PC) médio foi 52,09 cm (variação entre 48,5 e 56,0 cm); a mediana foi $52,00 \mathrm{~cm}$ e o desvio padrão 1,44. Considerando-se a distribuição segundo o sexo, observou-se que no sexo masculino, todos apresentaram PC dentro de limites normais. No sexo feminino, detectouse microcefalia em uma e macrocefalia em 4 meninas.

O diâmetro bi-auricular (BA) médio foi 29,30 cm (variação entre 26,5 e $32,0 \mathrm{~cm}$ ); a mediana foi de 29,00 cm e desvio padrão foi1,30. O diâmetro antero-posterior (AP) médio foi $30,04 \mathrm{~cm}$ (variação entre 26,0 e $33,0 \mathrm{~cm}$ ); a mediana foi de $30,00 \mathrm{~cm}$ e o desvio padrão foi 1,39 .

Na avaliação da fala, apresentaram atraso na aquisição, 7 (5,64\%) escolares, caracterizandose por dislalia por troca e supressão em 1; dislalia por troca em 2 e dislalia por supressão em 4 .

Não foi observada alteração de força muscular nos membros. Um escolar apresentou paresia facial central à direita (Caso 18).

$\mathrm{Na}$ avaliação do tono muscular, apresentaram leve hipotonia nos membros, 15 (12,09\%) escolares. Na manobra de balanço passivo porém, foi observado, em $81(65,32 \%)$ escolares, leve aumento da amplitude do movimento nos membros superiores, levando à execução mais lenta dessa manobra. Considerando a distribuição da amplitude aumentada na manobra do balanço passivo segundo o sexo, não houve diferença significativa $\left(\chi^{2}=0,96 ; \mathrm{p}=0,32\right)$ (Tabela 2).

Os reflexos profundos foram normoativos em 53 (42,74\%); hipoativos em 60 (48,38\%); vivos em $10(8,06 \%)$, e hiperativos em um $(0,80 \%)$. Observou-se assimetria isolada de reflexo aquiliano em um $(0,80 \%)$ (Caso 3$)$.

O reflexo cutâneo plantar foi em flexão em todos. Observou-se clono bilateral facilmente esgotável em dois $(1,61 \%)$ escolares.

Na manobra de coordenação apendicular index-index verificou-se leve tremor bilateral em membros superiores em $12(9,67 \%)$ escolares, sendo do sexo masculino, 10. Houve diferença significativa quanto ao sexo $\left(\alpha^{2}=4,36 ; \mathrm{p}=0,036\right)$.

O leve tremor bilateral esteve associado ao aumento da amplitude do balanço passivo dos membros superiores em 9 (7,25\%) e foram normotônicos três escolares (Casos 117, 122 e 127). Não houve associação significativa entre o tremor e o balanço passivo aumentado $\left(\alpha^{2}=0,55 ; \mathrm{p}=0,45\right)$.

$\mathrm{Na}$ avaliação dos nervos cranianos, foi observado em 1 (0,80\%) escolar (Caso 18), leve estrabismo convergente não paralítico à direita, associado a paresia facial central direita, caracterizada por apagamento do sulco nasogeniano à direita e discreto desvio de rima bucal para a esquerda.

\section{DISCUSSÃO}

Neste estudo, avaliando alunos de classe comum de $1^{\text {a }}$ série do ensino fundamental, anormalidades de respostas em pelo menos uma das provas do ENT foram observadas em 29,83\% dos escolares. Os achados foram isolados, não foi possível identificar síndromes neurológicas, nem tiveram valor preditivo de localização, com exceção de um escolar, em que foi possível identificar a síndrome específica de liberação do trato córtico-espinal nos membros inferiores. 
O ENT utiliza técnica semiológica voltada para detectar lesões específicas de núcleos, tratos ou nervos. São as anormalidades neurológicas maiores (hard signs) ou síndromes neurológicas, que tem valor preditivo de localização ${ }^{12}$.

Essa técnica semiológica permite observar também os sinais neurológicos menores (soft signs). Embora anormais, são assim chamados por não terem tal valor preditivo de localização ${ }^{12}$. Esse termo tem sido utilizado para descrever várias anormalidades no exame neurológico, que se acredita não sejam parte de síndrome neurológica bem definida ${ }^{13-15}$. O significado clínico desses sinais não é claro e eles foram considerados como indicadores de lesão cerebral inespecífica. Refletem disfunção nas áreas de coordenação motora, função sensorial integrativa e organização de tarefas motoras complexas.

Estão incluídos no conceito de sinais neurológicos menores, fenômenos como a astereognosia, reflexos primitivos, disdiadococcinesia, movimentos em espelho, extinção. São considerados também como exemplos de sinais neurológicos menores, movimentos involuntários, incoordenação e anormalidades sensoriais como a astereognosia e agrafoestesia. Nas últimas décadas têm sido apresentadas avaliações padronizadas dos sinais neurológicos menores ${ }^{14}$.

Alguns autores sugerem que os sinais neurológicos menores na infância são marcadores transitórios de imaturidade do neurodesenvolvimento, claramente relacionados à idade cronológica ${ }^{16-}$ 17. Apesar das mudanças com a idade cronológica, outros autores sugerem que a presença desses sinais menores na infância, são preditivos de execução motora pobre na adolescência ou mais tarde ${ }^{13,18}$.

Para a maioria dos escolares desta pesquisa, as alterações encontradas na semiologia neurológica foram compatíveis com as definições dos sinais neurológicos menores (soft signs) ${ }^{12-15}$. Não tiveram valor preditivo de localização e descreveram várias anormalidades no exame neurológico, que se acredita não sejam parte de síndrome neurológica bem definida.

Neurologistas infantis que tem se dedicado ao estudo de crianças com dificuldade para a aprendizagem, afirmam que a semiologia neurológica através do ENT não deve encontrar alterações significativas, quando se investigam crianças com dificuldades escolares devidas a distúrbios psiconeurológicos ${ }^{2}$.

Na avaliação de 100 escolares da $1^{\mathrm{a}}$ série do $1^{\mathrm{o}}$ grau previamente classificados em dois grupos, com bom rendimento escolar e com dificuldades escolares, utilizando essa mesma semiologia, não se observaram alterações ao ENT capazes de distinguir os grupos ${ }^{19}$.

Comparando dois grupos de crianças com dificuldades escolares, em que um dos grupos apresentava alterações neurológicas orgânicas associadas, outro autor nacional ${ }^{9}$ encontrou semiologia neurológica alterada em $58,00 \%$ e $90,90 \%$ respectivamente, predominando a hipotonia muscular leve em $54,5 \%$ e o tremor fino de extremidades em $45,4 \%$; em sua maioria, cada exame apresentou 2 ou 3 alterações. Apesar de serem a hipotonia muscular e o tremor fino de extremidades os achados mais frequentes nessas pesquisas, a diferença se faz na frequência com que são encontradas entre os escolares comuns $(9,67 \%)$ e aqueles com dificuldade escolar $(58,00 \%$ e $90,00 \%)$.

O atraso na aquisição da fala foi observado em $8,68 \%$ dos escolares avaliados nesta pesquisa. Esta frequência foi semelhante aos resultados encontrados com crianças que apresentavam dificuldade escolar $^{19}$, quando foram observadas dislalias em $4 \%$.

Crianças portadoras de deficiência mental ${ }^{21}$, apresentaram o exame de fala alterado em $33,1 \%$, sendo mais frequente a dislalia por troca e supressão de fonemas. Não houve diferença quanto à alteração na fala segundo os graus de deficiência mental. Crianças epilépticas avaliadas através dessa semiologia, apresentaram alteração de fala em 52\%, sendo mais frequente a dislalia por troca e supressão, na faixa etária de 3 a $4 \operatorname{anos}^{22}$. Crianças sem dificuldade escolar avaliadas com a mesma semiologia da fala, não apresentaram alteração aos $7 \operatorname{anos}^{20}$, não havendo diferença nos resultados devidas ao sexo ou à proveniência escolar. A partir dos 4 anos de idade cronológica as crianças apresentavam fala normal. 
Considerando dados da literatura, a fala não deve apresentar alterações aos 7 anos de idade. Entretanto essa alteração foi encontrada nessa faixa etária, em escolares com dificuldade ou em deficientes mentais. Os resultados encontrados na avaliação da fala nessa pesquisa, levam a indícios de que os escolares que apresentaram atraso na aquisição, principalmente aqueles com dislalias por troca, deverão ser acompanhados anualmente quanto à escolaridade e se necessário, ter sua avaliação neurológica complementada por equipe interdisciplinar, para excluir a deficiência mental ou distúrbio de aprendizagem.

Na avaliação do tono muscular pela movimentação passiva, palpação muscular e balanço passivo, verificou-se nesta pesquisa que apresentaram leve hipotonia nos membros $12,09 \%$ dos escolares e não foi observada hipertonia muscular.

Utilizando estas mesmas manobras de palpação e balanço passivo em crianças sem dificuldade escolar, diferentes autores nacionais têm observado hipotonia muscular numa frequência variando entre $3,5 \%{ }^{20} \mathrm{e} 22 \%{ }^{19}$, resultados entre os quais encontram-se os desta pesquisa. $\mathrm{O}$ achado de hipertonia muscular observado por um dos autores ${ }^{19}$, foi atribuído à resistência voluntária às manobras de avaliação de tono muscular.

Considerando-se apenas a manobra de balanço passivo porém, foi observada nesta pesquisa uma diferença qualitativa em $65,32 \%$ dos escolares, caracterizada por leve aumento da amplitude do movimento nos membros superiores, levando a uma execução mais lenta dessa manobra. Não houve diferença significativa segundo o sexo.

Avaliando crianças com dificuldade escolar ${ }^{19}$, foi referido que o único aspecto do ENT que se mostrava patológico era o tono muscular, sendo encontrado maior número de hipotônicos no grupo com dificuldade escolar (42\%), comparados com o grupo com bom rendimento escolar (22\%). Embora os resultados fossem diferentes, encontrando quase o dobro de hipotônicos, não houve diferença estatisticamente significativa, sendo inferido que tal verificação é insuficiente para distinguir os dois grupos.

Neste estudo, os reflexos profundos foram normoativos ou vivos em 50,80\% dos escolares, hipoativos em $48,38 \%$ e exaltados em $0,80 \%$. Observou-se assimetria de reflexo aquiliano em $0,80 \%$. Esses achados foram isolados, com exceção de um escolar.

Avaliando reflexos profundos em crianças sem dificuldade escolar ${ }^{20}$, os reflexos foram normoativos em $94,5 \%$ e hiporreflexia em 5,5\% dos sujeitos, sempre com respostas simétricas.

Na manobra de coordenação apendicular index-index verificou-se, nesta pesquisa, leve tremor bilateral em membros superiores em $8,87 \%$ dos escolares, havendo predomínio no sexo masculino.

$\mathrm{Na}$ avaliação da coordenação apendicular de escolares com dificuldade escolar ${ }^{6}$, foi observado leve tremor de extremidades em $16,6 \%$ ou ainda a função coordenação apendicular foi a que mais discriminou os dois grupos com e sem dificuldade escolar, havendo diferença significativa ${ }^{19}$.

Naqueles escolares que apresentaram 2 sinais neurológicos, presentes em 4,83\% das crianças, os achados foram compatíveis com alteração difusa do SNC, caracterizadas pela associação de alterações do perímetro craniano, da fala ou clono, associados a leve tremor ou leve hipotonia muscular.

Esses sinais descreveram várias anormalidades no exame neurológico, que se acredita não sejam parte de síndrome neurológica bem definida. O significado clínico desses sinais não foi claro e eles foram considerados como indicadores de lesão cerebral inespecífica. Essa semiologia poderia tornar mais claro o comprometimento neurológico, se complementada pela avaliação de função nas áreas de coordenação motora, função sensorial integrativa e organização de tarefas motoras mais complexas. Outra opção seria o acompanhamento neurológico longitudinal desses escolares, verificando-se como se manifestam essas discretas alterações em faixa etária maior.

Avaliando longitudinalmente crianças hiperativas ${ }^{23}$, foi observado que as alterações neurológicas encontradas aos 6-7 anos de idade desapareceram em grande parte nas avaliações 
subsequentes aos 10-13 anos. Esses achados questionam a estabilidade das anormalidades neurológicas a longo prazo.

No que se refere ao grupo avaliado nesta pesquisa, todos frequentando classe normal de $1^{\mathrm{a}}$ série primária, verificou-se que nem todos apresentaram semiologia neurológica normal. Decidir sobre a natureza exata das alterações encontradas não é tarefa fácil.

As alterações na fala, nas medidas de crânio, incoordenação, hiperatividade ou mais que uma alteração na semiologia neurológica, apesar de não constituírem síndrome neurológica específica, podem ser encontradas em crianças com distúrbios e dificuldades escolares, retardo mental, etc.

$\mathrm{O}$ acompanhamento longitudinal anual quanto à escolaridade, semiologia neurológica e se necessário, a avaliação complementada por equipe interdisciplinar, poderão esclarecer esses achados.

Agradecimentos - Ao Serviço de Recursos Audiovisuais da Unicamp, pela infra-estrutura de informática utilizada na coleta de dados. À equipe técnica do GIADI (Grupo Interdisciplinar de Avaliação do Desenvolvimento Infantil), pela dedicação e entusiasmo no trabalho em conjunto. À psicologa Adriana Aparecida Ferrarezi, eficiente auxiliar de pesquisa. À Profa Dra Denise Yvonne J. Norato, pela orientação e colaboração constantes.

\section{REFERÊNCIAS}

1. Lefévre BH. Avaliação neuropsicológica da criança. In Diament A, Cypel S. Neurologia infantil. 3Ed, São Paulo Atheneu, 1996,83.

2. Rotta NT, Guardiola A. Distúrbios da aprendizagem. In Diament AJ, Cypel S. Neurologia Infantil. São Paulo: Atheneu, 1996:1062-1074.

3. Rotta NT. Aspectos neurológicos de los problemas de aprendizagem. Ann Neuropediatr Latinoamer 1988,1:11.

4. Weiss MLL. Psicopedagogia Clínica. Porto Alegre: Artes Médicas, 1992.

5. Coll C, Bolea E. As intenções educativas e os objetivos da educação escolar: alternativas e fundamentos psicológicos. In Coll C et al. Desenvolvimento psicológico e educação: psicologia da educação. Porto Alegre: Artes Médicas, 1996.

6. Tonelotto JMF. Atenção e sua relação com atitudes de crianças no contexto escolar. Tese de Doutorado. Faculdade de Ciências Médicas da Unicamp, Campinas, 1998

7. Romero JF. As relações sociais das crianças com dificuldades de aprendizagem. In Coll, C et al. Desenvolvimento psicológico e educação: necessidades educativas especiais e aprendizagem escolar psicologia da educação, vol 3. Porto Alegre: Artes Médicas, 1995.

8. Ciasca SM. Diagnóstico nos distúrbios de aprendizagem em crianças: análise de uma prática interdisciplinar. Dissertação de Mestrado, Instituto de Psicologia da USP. São Paulo, 1990.

9. Ciasca SM. Distúrbios e dificuldades de aprendizagem em crianças: análise do diagnóstico interdisciplinar. Tese de Doutorado. Faculdade de Ciências Médicas da Unicamp. Campinas, 1994.

10. Lefèvre AB. O exame neurológico da criança. In Tolosa APM, Canelas HM (eds). Propedêutica neurológica. São Paulo: Sarvier 1972.

11. Diament AJ, Rodrigues FW. Cephalic measures in normal preschool children 3 to 7 years of age. Arq Neuropsiquiatr 1976;34:325-329.

12. Keenam E, O’Donnell C, Sinanan K, O'Callaghan E. Severity of alchool dependence and its relationship to neurological soft signs, neuropsychological impairment and family history. Acta Psychiatr Scand 1997;95:272-276.

13. Shaffer D; Schonfeld IS; O'Connor P. Neurological soft signs and their relationship to psychatric disorder and intelligence in childhood and adolescence. Arch Gen Psychiatry 1985;42:342-351.

14. Pine DS, Scott MR, Busner C et al. Psychometrics of neurological soft signs. J Am Acad Child Adolesc Psychiatry 1996;35:509-515.

15. Kennard MA. Value of equivocal signs in neurologic diagnosis. Neurology 1960;10:753-764.

16. Mikkelsen EJ, Brown GL, Minichiello MD, Millican FK, Rapoport JL. Neurologic status in hyperative, enuretic and normal boys. J Am Acad Child Adolesc Psychiatry 1982;21:75-81.

17. Vitiello B, Ricciuti A, Stoff D, Behar D, Denckla MB. Reliability of subtle (soft) neurological signs in children. J Am Acad Child Adolesc Psychiatry, 1989,28:749-753.

18. Hertzig MD. Stability and change in non focal neurological signs. J Am Acad Child Adolesc Psychiatry 1982;21:231-236.

19. Rotta NT. Avaliação neurológica evolutiva, eletrencefalográfica e psicológica em crianças com rendimento escolar deficiente. Tese de Docência-Livre, Faculdade de Medicina da UFRGS. Porto Alegre, 1975.

20. Bachiega MCM. Exame neurológico evolutivo da criança normal de 3 a 7 anos de idade: contribuição para a avaliação da fidedignidade das provas. Dissertação de Mestrado. Faculdade de Medicina da USP. São Paulo, 1979.

21. Gonçalves VMG. Exame neurológico evolutivo em deficientes mentais. Dissertação de Mestrado, Facudade de Medicina da USP. São Paulo, 1984.

22. Elkis, LC. Estudo do desenvolvimento neurológico em crianças epilépticas. Dissertação de Mestrado, Faculdade de Medicina da USP. São Paulo, 1982.

23. Gillberg C, Groth J. Children with preschool minor neurodevelopmental disorders: neurodevelopmental profiles at age 13. Dev Med Child Neurol. 1989;31:14-24. 